This item was submitted to Loughborough's Research Repository by the author.

Items in Figshare are protected by copyright, with all rights reserved, unless otherwise indicated.

\title{
Superconducting gap, normal state pseudogap, and tunneling spectra of bosonic and cuprate superconductors
}

PLEASE CITE THE PUBLISHED VERSION

PUBLISHER

(C) American Physical Society

\section{VERSION}

VoR (Version of Record)

\section{LICENCE}

CC BY-NC-ND 4.0

\section{REPOSITORY RECORD}

Alexandrov, A.S., and Joanne Beanland. 2019. "Superconducting Gap, Normal State Pseudogap, and Tunneling Spectra of Bosonic and Cuprate Superconductors". figshare. https://hdl.handle.net/2134/5848. 
This item was submitted to Loughborough's Institutional Repository (https://dspace.lboro.ac.uk/) by the author and is made available under the following Creative Commons Licence conditions.

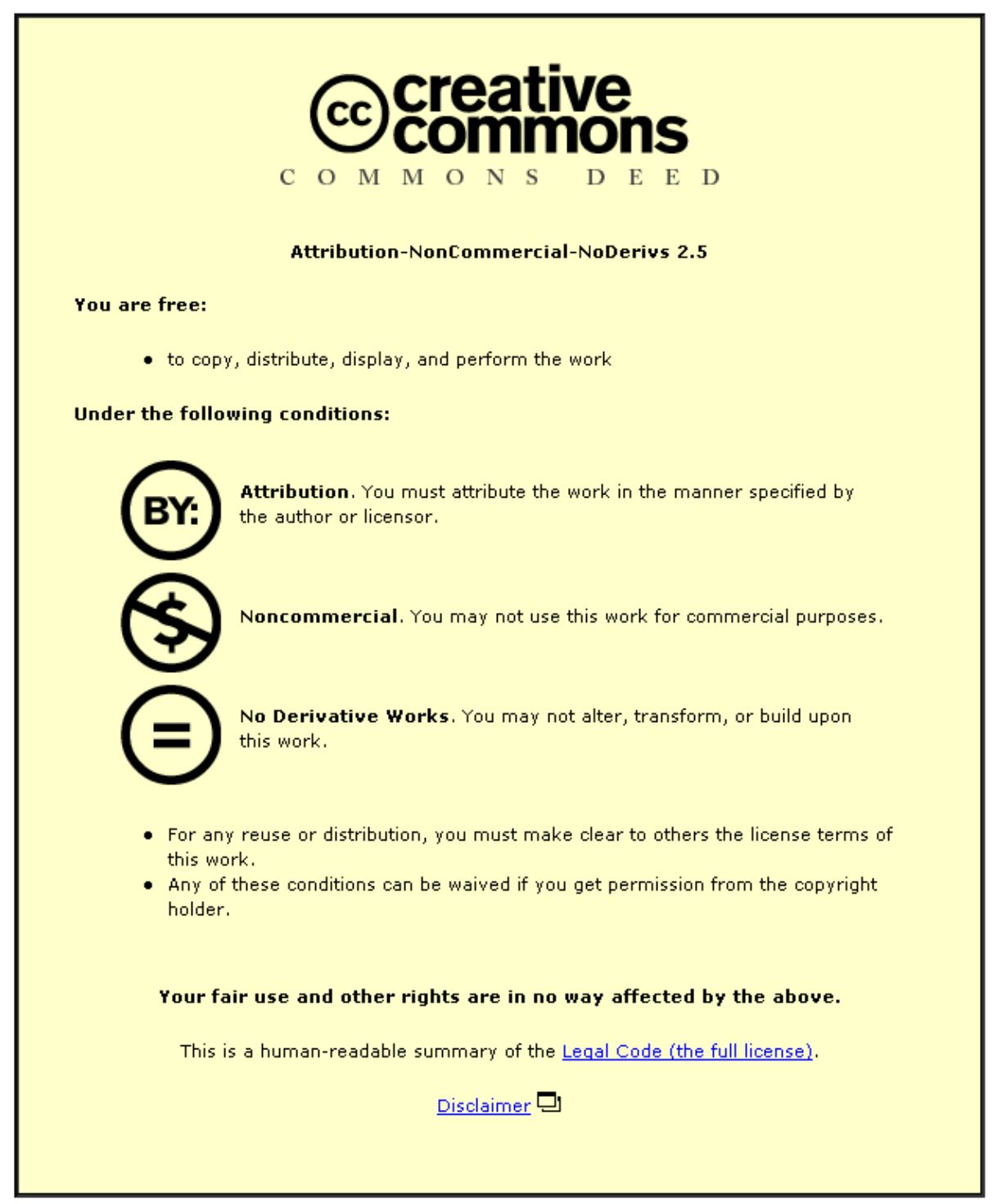

For the full text of this licence, please go to: http://creativecommons.org/licenses/by-nc-nd/2.5/ 


\title{
Superconducting Gap, Normal State Pseudogap, and Tunneling Spectra of Bosonic and Cuprate Superconductors
}

\author{
A. S. Alexandrov and J. Beanland \\ Department of Physics, Loughborough University, Loughborough LE11 3TU, United Kingdom
}

(Received 21 October 2009; published 12 January 2010)

\begin{abstract}
We develop a theory of normal metal-superconductor and superconductor tunneling in "bosonic" superconductors with strong attractive correlations taking into account coherence effects in single-particle excitation spectrum and disorder. The theory accounts for the existence of two energy scales, their temperature and doping dependencies, asymmetry, and inhomogeneity of tunneling spectra of underdoped cuprate superconductors.
\end{abstract}

DOI: 10.1103/PhysRevLett.104.026401

Soon after the discovery of high- $T_{c}$ superconductivity [1], a number of tunneling, photoemission, optical, nuclear spin relaxation, and electron-energy-loss spectroscopies discovered an anomalous large gap in cuprate superconductors existing well above the superconducting critical temperature, $T_{c}$. The gap, now known as the pseudogap, was originally assigned [2] to the binding energy of real-space preformed hole pairs-small bipolaronsbound by a strong electron-phonon interaction (EPI). Since then, alternative explanations of the pseudogap have been proposed, including preformed Cooper pairs [3], inhomogeneous charge distributions containing holerich and hole-poor domains [4], or competing quantum phase transitions [5].

Present-day scanning tunneling (STS) [6-8], intrinsic tunneling [9], and angle-resolved photoemission (ARPES) [5] spectroscopies have offered a tremendous advance into the understanding of the pseudogap phenomenon in cuprates and some related compounds. Both extrinsic (see [6,8] and references therein) and intrinsic [9] tunneling as well as high-resolution ARPES [5] have found another energy scale, reminiscent of a BCS-like "superconducting" gap that opens at $T_{c}$ accompanied by the appearance of Bogoliubov-like quasiparticles [5] around the node. Earlier experiments with a time-resolved pump probe demonstrated two distinct gaps, one a temperature independent pseudogap and the other a BCS-like gap [10]. Also, Andreev reflection experiments revealed a much smaller gap edge than the bias at the tunneling conductance maxima in a few underdoped cuprates [11]. Another remarkable observation is the spatial nanoscale inhomogeneity of the pseudogap observed with STS [6-8] and presumably related to an unavoidable disorder in doped cuprates, Fig. 1(a). Essentially, the doping and magnetic field dependence of the superconducting gap compared with the pseudogap and their different real-space profiles have prompted an opinion that the pseudogap is detrimental to superconductivity and connected to a quantum critical point rather than to preformed Cooper pairs [9]. Nevertheless, without a detailed microscopic theory that can describe highly unusual tunneling and ARPES spectra,
PACS numbers: 71.38. $-\mathrm{k}, 72.15 . J f, 74.40 .-\mathrm{n}, 74.72 .-\mathrm{h}$

the relationship between the pseudogap and the superconducting gap remains a mystery [5]. It has become increasingly likely that NS and SS tunneling spectra of underdoped cuprates do not agree with the simplest BCS spectra, neither $s$ wave nor $d$ wave. Apart from the almost temperature independent pseudogap, $\Delta_{p}$ with $2 \Delta_{p} / k_{B} T_{c}$ often many times larger than the BCS ratio $(\approx 3.5)$, there is an asymmetry in NS tunneling, Fig. 1. The integrated conductance for the negative bias is larger than for the positive bias in many samples. The van Hove singularity (vHs) of the density of states (DOS) is ruled out as a possible origin of the asymmetry since it is absent in the momentumintegrated photoemission, Fig. 1(b). The asymmetry is expected for conventional semiconductors or MottHubbard insulators $\left[I_{\mathrm{ns}}(-\infty) / I_{\mathrm{ns}}(+\infty)=(1-p) / 2 p\right]$, but neither of them accounts for its magnitude, Fig. 1, if disorder and matrix elements are not considered. Here, we

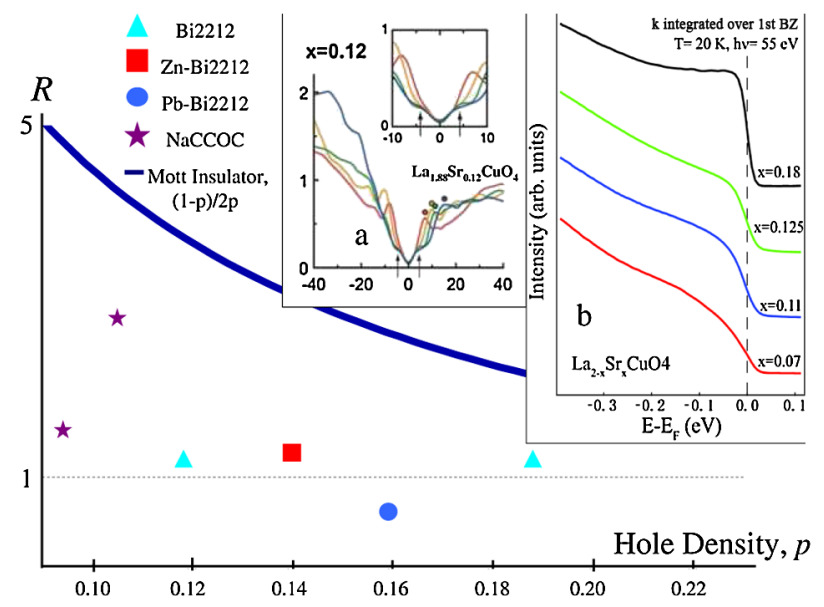

FIG. 1 (color online). Ratio, $R=I_{\mathrm{ns}}(-100) / I_{\mathrm{ns}}(100)$, of the negative-bias NS tunneling conductance to the positive bias conductance [25] integrated from $0 \mathrm{meV}$ to $\mp 100 \mathrm{meV}$, respectively, for a few cuprate superconductors in a wide range of atomic hole density, $p$. Inset (a) atomically resolved asymmetric STS spectra of $\mathrm{La}_{1.88} \mathrm{Sr}_{0.12} \mathrm{CuO}_{4}$ at $4.2 \mathrm{~K}$ acquired at different points of the scan area [8]; (b) momentum-integrated photoemission showing no vHs of DOS [26]. 
develop the theory of NS and SS tunneling in bosonic superconductors with strong attractive correlations [12] by taking into account disorder and coherence effects in a single-particle excitation spectrum. Our theory accounts for peculiarities in extrinsic and intrinsic tunneling in underdoped cuprate superconductors.

Recent Monte Carlo calculations show that high- $T_{c}$ superconductivity cannot be explained by the simplest repulsive Hubbard model [13] so that one has to extend the model to get some superconducting order [14]. On the other hand, even a moderate EPI significantly increases the superconducting condensation energy [15] stabilizing mobile small bipolarons [16,17], as anticipated for strongly correlated electrons in highly polarizable ionic lattices [12]. Real-space pairs, whatever the pairing interaction is, can be described as a charged Bose liquid on a lattice if the carrier density is relatively small [12]. The superfluid state of such a liquid is the true Bose-Einstein condensate (BEC), rather than a coherent state of overlapping Cooper pairs. Single-particle excitations of the liquid are thermally excited single polarons propagating in a doped insulator band or are localized by impurities. Different from the BCS case, their negative chemical potential, $\mu$, is found outside the band by about half of the bipolaron binding energy, $\Delta_{p}$, both in the superconducting and normal states [12]. Here, in the superconducting state $\left(T<T_{c}\right)$, following Ref. [18], we take into account that polarons interact with the condensate via the same potential that binds the carriers so that the single-particle Hamiltonian in the Bogoliubov approximation is

$$
H_{0}=\sum_{\nu}\left[\xi_{\nu} p_{\nu}^{\dagger} p_{\nu}+\left(\frac{1}{2} \Delta_{c \nu} p_{\bar{\nu}}^{\dagger} p_{\nu}^{\dagger}+\text { H.c. }\right)\right],
$$

where $\xi_{\nu}=E_{\nu}-\mu, E_{\nu}$ is the normal-state single-polaron energy spectrum in the crystal field and disorder potentials renormalized by EPI and spin fluctuations, and $\Delta_{c \nu}=$ $-\Delta_{c \bar{\nu}}$ is the coherent potential proportional to the square root of the condensate density, $\Delta_{c} \propto \sqrt{n_{c}(T)}$. The operators $p_{\nu}^{\dagger}$ and $p_{\bar{\nu}}^{\dagger}$ create a polaron in the single-particle quantum state $\nu$ and in the time-reversed state $\bar{\nu}$, respectively. As in the BCS case, the single-quasiparticle energy spectrum, $\epsilon_{\nu}$, is found using the Bogoliubov transformation, $p_{\nu}=$ $u_{\nu} \alpha_{\nu}+v_{\nu} \beta_{\nu}^{\dagger}, p_{\bar{\nu}}=u_{\nu} \beta_{\nu}-v_{\nu} \alpha_{\nu}^{\dagger}, \epsilon_{\nu}=\left[\xi_{\nu}^{2}+\Delta_{c \nu}^{2}\right]^{1 / 2}$, with $u_{\nu}^{2}, v_{\nu}^{2}=\left(1 \pm \xi_{\nu} / \epsilon_{\nu}\right) / 2$. This spectrum is different from the BCS quasiparticles because the chemical poten- tial is negative with respect to the bottom of the singleparticle band, $\mu=-\Delta_{p}$. A single-particle gap, $\Delta$, is defined as the minimum of $\epsilon_{\nu}$. Without disorder, for a pointlike pairing potential with the $s$ wave coherent gap, $\Delta_{c \mathbf{k}} \approx \Delta_{c}$, one has [18] $\Delta(T)=\left[\Delta_{p}^{2}+\Delta_{c}(T)^{2}\right]^{1 / 2}$. The full gap varies with temperature from $\Delta(0)=\left[\Delta_{p}^{2}+\right.$ $\left.\Delta_{c}(0)^{2}\right]^{1 / 2}$ at zero temperature down to the temperature independent $\Delta=\Delta_{p}$ above $T_{c}$, which qualitatively describes some earlier and more recent [9] observations including the Andreev reflection in cuprates (see [18] and references therein). The NS and SS tunneling transitions are described with the tunneling Hamiltonians, $H_{\mathrm{ns}}$ and $H_{\mathrm{ss}}$, respectively [19], which are perturbations

$$
\begin{aligned}
H_{\mathrm{ns}}= & P \sum_{\nu \nu^{\prime}} p_{\nu^{\prime}}^{\dagger} c_{\nu}+B N^{-1 / 2} \sum_{\nu \nu^{\prime} \eta^{\prime}} b_{\eta^{\prime}}^{\dagger} p_{\bar{\nu}^{\prime}} c_{\nu}+\text { H.c., } \\
H_{\mathrm{ss}}= & P \sum_{\nu, \nu^{\prime}} p_{\nu^{\prime}}^{\dagger} p_{\nu}+B N^{-1 / 2} \\
& \times \sum_{\nu \nu^{\prime}}\left(\sum_{\eta} p_{\nu^{\prime}}^{\dagger} p_{\bar{\nu}}^{\dagger} b_{\eta}+\sum_{\eta^{\prime}} b_{\eta^{\prime}}^{\dagger} p_{\nu} p_{\bar{\nu}^{\prime}}\right)+\text { H.c. }
\end{aligned}
$$

Here, $c_{\nu}, p_{\nu^{\prime}}$, and $b_{\eta^{\prime}}$ annihilate a carrier in the normal metal, a single polaron, and a composed boson in the bosonic superconductor, respectively, $N$ is the number of unit cells. Generally, the tunneling matrix elements with $(B)$ and without $(P)$ involvement of the composed boson are different, $B \geq P$, because the presence of an additional hole lowers the tunneling barrier for an injection of the electron [19]. Applying the Bogoliubov transformation to Eqs. (2) and the standard perturbation theory yields the following current-voltage characteristics:

$$
\begin{aligned}
I_{\mathrm{ns}}(V)= & \frac{2 \pi e P^{2}}{\hbar} \sum_{\nu \nu^{\prime}}\left[u_{\nu^{\prime}}^{2}\left(F_{\nu}-f_{\nu^{\prime}}\right) \delta\left(\xi_{\nu}+e V-\epsilon_{\nu^{\prime}}\right)\right. \\
& \left.+v_{\nu^{\prime}}^{2}\left(F_{\nu}+f_{\nu^{\prime}}-1\right) \delta\left(\xi_{\nu}+e V+\epsilon_{\nu^{\prime}}\right)\right] \\
& +\frac{2 \pi e B^{2}}{\hbar} \sum_{\nu \nu^{\prime}}\left\{u_{\nu^{\prime}}^{2}\left[F_{\nu} f_{\nu^{\prime}}-(x / 2)\left(1-F_{\nu}-f_{\nu^{\prime}}\right)\right]\right. \\
& \times \delta\left(\xi_{\nu}+e V+\epsilon_{\nu^{\prime}}\right)+v_{\nu^{\prime}}^{2}\left[F_{\nu}\left(1-f_{\nu^{\prime}}\right)\right. \\
& \left.\left.+(x / 2)\left(F_{\nu}-f_{\nu^{\prime}}\right)\right] \delta\left(\xi_{\nu}+e V-\epsilon_{\nu^{\prime}}\right)\right\},
\end{aligned}
$$

where $\quad F_{\nu}=1 /\left[\exp \left(\xi_{\nu} / k_{B} T\right)+1\right], \quad f_{\nu^{\prime}}=$ $1 /\left[\exp \left(\epsilon_{\nu^{\prime}} / k_{B} T\right)+1\right]$ are distribution functions of carriers in the normal metal and single quasiparticles, respectively, and $x / 2$ is the atomic density of composed bosons in the superconductor, and

$$
\begin{aligned}
I_{\mathrm{ss}}(V)= & \frac{2 \pi e P^{2}}{\hbar} \sum_{\nu \nu^{\prime}}\left\{\left(u_{\nu}^{2} u_{\nu^{\prime}}^{2}+v_{\nu}^{2} v_{\nu^{\prime}}^{2}\right)\left(f_{\nu}-f_{\nu^{\prime}}\right) \delta\left(\epsilon_{\nu}+e V-\epsilon_{\nu^{\prime}}\right)+u_{\nu}^{2} v_{\nu^{\prime}}^{2}\left(f_{\nu}+f_{\nu^{\prime}}-1\right)\right. \\
& \left.\times\left[\delta\left(\epsilon_{\nu}+e V+\epsilon_{\nu^{\prime}}\right)-\delta\left(\epsilon_{\nu}-e V+\epsilon_{\nu^{\prime}}\right)\right]\right\}+\frac{2 \pi e B^{2}}{\hbar} \sum_{\nu \nu^{\prime}}\left\{u_{\nu}^{2} u_{\nu^{\prime}}^{2}\left[\left(1-f_{\nu}-f_{\nu^{\prime}}\right) x / 2-f_{\nu} f_{\nu^{\prime}}\right]\right. \\
& +v_{\nu}^{2} v_{\nu^{\prime}}^{2}\left[\left(1-f_{\nu}-f_{\nu^{\prime}}\right) x / 2+\left(1-f_{\nu}\right)\left(1-f_{\nu^{\prime}}\right)\right]\left[\delta\left(\epsilon_{\nu}-e V+\epsilon_{\nu^{\prime}}\right)-\delta\left(\epsilon_{\nu}+e V+\epsilon_{\nu^{\prime}}\right)\right] \\
& \left.+2 u_{\nu}^{2} v_{\nu^{\prime}}^{2}\left[\left(f_{\nu^{\prime}}-f_{\nu}\right) x / 2-f_{\nu}\left(1-f_{\nu^{\prime}}\right)\right]\left[\delta\left(\epsilon_{\nu}+e V-\epsilon_{\nu^{\prime}}\right)-\delta\left(\epsilon_{\nu}-e V-\epsilon_{\nu^{\prime}}\right)\right]\right\},
\end{aligned}
$$


where $V$ is the voltage drop across the junction. For more transparency, we neglect the boson energy dispersion in Eqs. (3) and (4), assuming that bosons are sufficiently heavy, so their bandwidth is relatively small. Here, we adopt the "LDA + GTB" band structure with impurity bandtails near $(\pi / 2, \pi / 2)$ of the Brillouin zone, Fig. 2(a), which explains the charge-transfer gap, $E_{\mathrm{ct}}$, the nodes and sharp "quasiparticle" peaks, and the high-energy "waterfall" seen in ARPES [20]. The chemical potential is found in the single-particle bandtail within the charge-transfer gap at the bipolaron mobility edge, Fig. 2(b), in agreement with the SNS tunneling experiments [21]. Such a band structure explains an insulating-like low temperature normal-state resistivity as well as many other unusual properties of underdoped cuprates [12]. If the characteristic bandtail width of DOS, $\Gamma$, is sufficiently large compared with the coherent gap, $\Gamma \gtrsim \Delta_{c \nu}$, one can factorize the quasiparticle DOS as $\rho(E) \equiv \sum_{\nu} \delta\left(E-\epsilon_{\nu}\right) \approx$ $\left[\rho_{n}(E)+\rho_{n}(-E)\right] \rho_{s}(E)$ for any symmetry of the coherent gap. Here, $\rho_{n}(E)$ is the normal-state DOS of the doped insulator with the bandtail, Fig. 2(a), and $\rho_{s}(E)=$ $E / \sqrt{E^{2}-\Delta_{c}^{2}}$ for the $s$ wave gap, or $\rho_{s}(E)=(2 / \pi)[\Theta(1-$ $\left.\left.E / \Delta_{0}\right) E K\left(E / \Delta_{0}\right) / \Delta_{0}+\Theta\left(E / \Delta_{0}-1\right) K\left(\Delta_{0} / E\right)\right]$ for a $d$ wave gap, $\Delta_{c \nu}=\Delta_{0} \cos (2 \phi)$ [22] [K(x) is the complete elliptic integral and $\phi$ is an angle along the constant energy contour]. One can neglect the energy dependence of the normal metal DOS. Then, differentiating Eq. (3) over the voltage and integrating yields the NS tunneling conductance $\sigma_{\mathrm{ns}}=d I_{\mathrm{ns}} / d V$ at zero temperature for the $d$ wave case,

$$
\begin{aligned}
\sigma_{\mathrm{ns}} \propto & A^{+} \rho_{s}(|e V|)\left[\rho_{n}(-e V)+\rho_{n}(e V)\right] \\
& +A^{-}\left[1-2 \cos ^{-1}\left(|e V| / \Delta_{0}\right)\right. \\
& \left.\times \Theta\left(1-|e V| / \Delta_{0}\right) / \pi\right]\left[\rho_{n}(-e V)-\rho_{n}(e V)\right],
\end{aligned}
$$

where $A^{ \pm}=1 \pm B^{2}[\Theta(-e V)+x / 2] / P^{2}$, and $\Theta(E)$ is the Heaviside step function. The theoretical conductance, Eq. (5), calculated with a model normal-state DOS, $\rho_{n}(E) / \rho_{b}=\left\{1+\tanh \left[\left(E-\Delta_{p}\right) / \Gamma\right]\right\} / 2$, and the $d$ wave superconducting DOS, $\rho_{s}(E)$, is shown in Fig. 3. Our
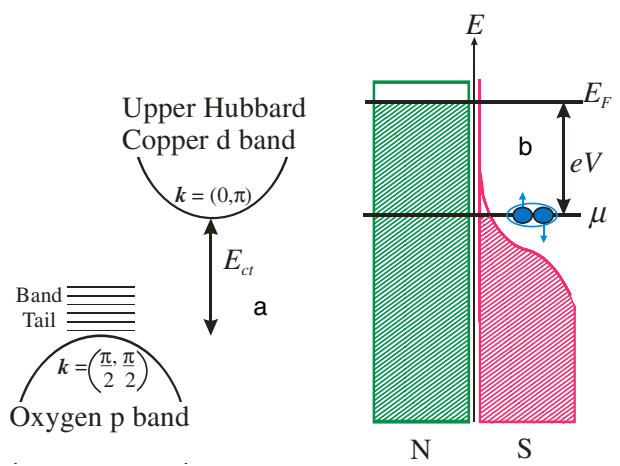

FIG. 2 (color online). LDA + GTB energy band structure of underdoped cuprates with the impurity localized states [20] shown as horizontal lines in (a); NS model densities of states (b) showing the bandtail in the bosonic superconductor. model $\rho_{n}(E)$ reflects the characteristic energy dependence of DOS in disordered doped insulators, which is a constant $\rho_{b}$ above the two-dimensional band edge and an exponent deep in the tail. Any particular choice of $\rho_{n}(E)$ and the model parameters can be made without affecting our conclusions as long as the characteristic features are reflected in this choice. Equation (5) captures all unusual signatures of the experimental tunneling conductance in underdoped cuprates, such as the low-energy coherent gap, the highenergy pseudogap, and the asymmetry. In the case of atomically resolved STS, one should replace the averaged DOS $\rho_{n}(E)$ in Eq. (5) with a local bandtail DOS $\rho_{n}(E, \mathbf{r})$, which depends on different points of the scan area $\mathbf{r}$ due to a nonuniform dopant distribution. As a result, the pseudogap shows nanoscale inhomogeneity, while the lowenergy coherent gap is spatially uniform, as observed [8], Fig. 1(a). Increasing doping level tends to diminish the bipolaron binding energy, $\Delta_{p}$, since the pairing potential becomes weaker due to a partial screening of EPI with lowfrequency phonons [23]. However, the coherent gap, $\Delta_{c}$, which is the product of the pairing potential and the square root of the carrier density [18], can remain about a constant or even increase with doping, as also observed [8].

In the case of the SS tunneling, we use Eq. (4) to address two unusual observations: a gapped conductance near and above $T_{c}$ and a negative excess resistance below $T_{c}[9,24]$. Equation (4) is grossly simplified in the normal state, where $\Delta_{c \nu}=0$,

$$
\begin{aligned}
I_{\mathrm{ss}}(V)= & \frac{2 \pi e P^{2}}{\hbar} \sum_{\nu \nu^{\prime}}\left(f_{\nu}-f_{\nu^{\prime}}\right) \delta\left(\xi_{\nu}+e V-\xi_{\nu^{\prime}}\right) \\
& +\frac{2 \pi e B^{2}}{\hbar} \sum_{\nu \nu^{\prime}}\left[\left(1-f_{\nu}-f_{\nu^{\prime}}\right) x / 2-f_{\nu} f_{\nu^{\prime}}\right] \\
& \times\left[\delta\left(\xi_{\nu}-e V+\xi_{\nu^{\prime}}\right)-\delta\left(\xi_{\nu}+e V+\xi_{\nu^{\prime}}\right)\right] .
\end{aligned}
$$

Near and above the transition but sufficiently below the pseudogap temperature, $T^{*} \equiv \Delta_{p} / k_{B}>T \gtrsim T_{c}$, and if the voltage is high enough, $e V \geqslant k_{B} T$, one can neglect temperature effects in Eq. (6) and approximate $f_{\nu}$ with the step

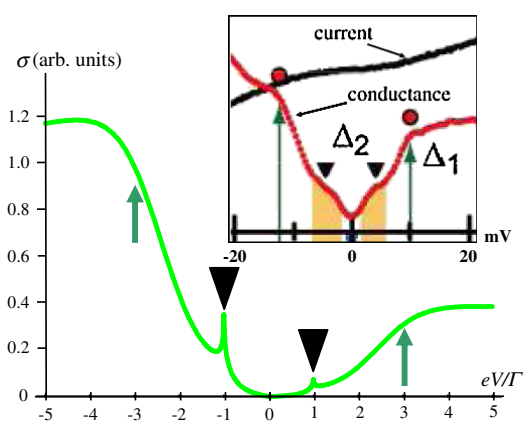

FIG. 3 (color online). Theoretical NS conductance, Eq. (5), for $\Delta_{0}=\Gamma, \Delta_{p}=2.7 \Gamma$ and $B=2.65 P$. The superconducting gap and the pseudogap are shown with triangles and arrows, respectively. Inset shows a representative STS spectrum of $\mathrm{La}_{2-x} \mathrm{Sr}_{x} \mathrm{CuO}_{4}$ with $x=0.12$ at $4.2 \mathrm{~K}$ [8]. 


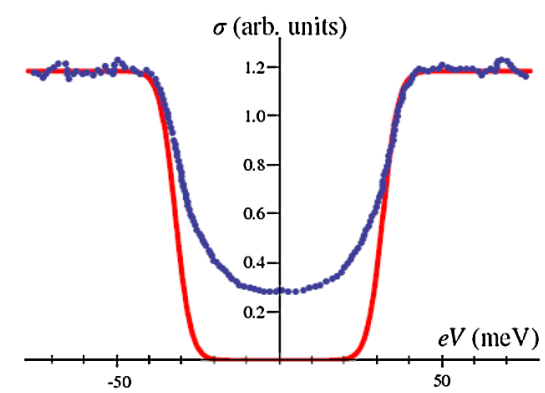

FIG. 4 (color online). Approximate normal-state tunneling conductance of bosonic superconductor (solid line) with $\Gamma=$ $3.2 \mathrm{meV}$ and $\Delta_{p}=16 \mathrm{meV}$ compared with the experimental conductance [9] (symbols) in mesas of $\mathrm{Bi}_{2} \mathrm{Sr}_{2} \mathrm{CaCu}_{2} \mathrm{O}_{8+\delta}\left(T_{c}=\right.$ $95 \mathrm{~K})$ at $T=87 \mathrm{~K}$.

function, $f_{\nu}=\Theta\left(-\xi_{\nu}\right)$. So using the model normal-state DOS yields

$$
\begin{aligned}
I_{\mathrm{ss}}(V) \propto & \frac{a^{2}}{2\left(a^{2}-1\right)}\left[\ln \frac{a^{2}\left(1+b^{2}\right)}{1+a^{2} b^{2}}-a^{-2} \ln \frac{a^{2}+b^{2}}{1+b^{2}}\right] \\
& +\frac{B^{2}(1+x / 2)}{P^{2}\left(a^{2} b^{4}-1\right)} \ln \frac{1+a^{2} b^{2}}{a\left(1+b^{2}\right)}+\frac{B^{2} x a^{2}}{2 P^{2}\left(a^{2}-b^{4}\right)} \\
& \times \ln \frac{a^{2}+b^{2}}{a\left(1+b^{2}\right)},
\end{aligned}
$$

where $a=\exp (|e V| / \Gamma)$ and $b=\exp \left(\Delta_{p} / \Gamma\right)$. When $b \gg$ 1 and $x$ is not too small, the first two terms on the righthand side of this equation are negligible. Hence, the tunneling matrix elements and doping have little effect on the shape of the current-voltage dependence. At sufficiently high voltages $e V \gtrsim k_{B} T$, the conductance [from $\sigma(V)=$ $d I_{\text {ss }} / d V$ with Eq. (7)] accounts for the gapped conductance in underdoped mesas of $\mathrm{Bi}_{2} \mathrm{Sr}_{2} \mathrm{CaCu}_{2} \mathrm{O}_{8+\delta}$ near and above $T_{c}$, as shown in Fig. 4. The finite temperature neglected in Eq. (7) accounts for some excess experimental conductance at low voltages in Fig. 4 compared with the theoretical conductance. The negative excess resistance below $T_{c}$ [24] can be explained by expanding Eqs. (4) and (6) in powers of $e V$ giving a zero-bias conductance. For low temperatures in the superconducting state, this is $\sigma_{s}(0) \propto$ $T^{-1} \int_{0}^{\infty} d \epsilon \rho_{s}(\epsilon)^{2} \cosh \left(\epsilon / 2 k_{B} T\right)^{-2}$, and in the normal state $\sigma_{n}(0) \propto T^{-1} \int_{-\infty}^{\infty} d \xi \rho_{n}(\xi)^{2} \cosh \left(\xi / 2 k_{B} T\right)^{-2}$. Estimating these integrals yields, respectively, $\sigma_{s}(0) \propto$ $T^{-1} \exp \left(-\Delta_{c} / k_{B} T\right)$ for the $s$ wave coherent gap, or $\sigma_{s}(0) \propto T^{2}$ for the $d$ wave gap, and $\sigma_{n}(0) \propto$ $T^{-1} \exp \left(-T^{*} / T\right)$. The latter expression is in excellent agreement with the temperature dependence of the mesa tunneling conductance above $T_{c}$ [24] (see also Ref. [23]). Extrapolating this expression to temperatures below $T_{c}$ yields the resistance ratio $R_{s} / R_{n} \propto \exp \left[\left(\Delta_{c} / k_{B}-T^{*}\right) / T\right]$ ( $s$ wave) or $R_{s} / R_{n} \propto \exp \left(-T^{*} / T\right) / T^{2}$ ( $d$ wave). Hence, in underdoped cuprates, where $T^{*}>\Delta_{c} / k_{B}$, the zero-bias tunneling resistance at temperatures below $T_{c}$ is smaller than the normal-state resistance extrapolated from above $T_{c}$ to the same temperatures (i.e., the negative excess resistance), as observed [24].
In summary, we have developed the theory of tunneling in bosonic superconductors by taking into account coherence effects in the single-quasiparticle energy spectrum, disorder and the realistic band structure of doped insulators. The theory accounts for the existence of two energy scales in the current-voltage NS and SS tunneling characteristics, their temperature and doping dependence, and for the asymmetry and inhomogeneity of NS tunneling spectra of underdoped cuprate superconductors.

We are grateful to Zhi-Xun Shen and Ruihua He for providing us with their momentum-integrated ARPES data, Fig. 1(b) and enlightening comments. We greatly appreciate valuable discussions with Ivan Bozovic, Kenjiro Gomes and Vladimir Krasnov and support of this work by EPSRC (UK) (Grant No. EP/H004483).

[1] J. G. Bednorz and K. A. Müller, Z. Phys. B 64, 189 (1986).

[2] A.S. Alexandrov and D. K. Ray, Philos. Mag. Lett. 63, 295 (1991).

[3] V. J. Emery and S. A. Kivelson, Nature (London) 374, 434 (1995).

[4] E. V.L. de Mello et al., Phys. Rev. B 66, 092504 (2002).

[5] W. S. Lee et al., Nature (London) 450, 81 (2007); R. H. He et al., Nature Phys. 5, 119 (2009).

[6] K. K. Gomes et al., Nature (London) 447, 569 (2007).

[7] J. Lee et al., Science 325, 1099 (2009).

[8] T. Kato et al., J. Phys. Soc. Jpn. 77, 054710 (2008).

[9] V. M. Krasnov, Phys. Rev. B 79, 214510 (2009).

[10] J. Demsar et al., Phys. Rev. Lett. 82, 4918 (1999).

[11] G. Deutscher, Nature (London) 397, 410 (1999).

[12] A.S. Alexandrov, Theory of Superconductivity: From Weak to Strong Coupling (IoP Publishing, Bristol, 2003).

[13] T. Aimi and M. Imada, J. Phys. Soc. Jpn. 76, 113708 (2007).

[14] D. Baeriswyl et al., New J. Phys. 11, 075010 (2009).

[15] T. M. Hardy et al., Phys. Rev. B 79, 212501 (2009).

[16] J. P. Hague et al., Phys. Rev. Lett. 98, 037002 (2007).

[17] J. Bonča and S. A. Trugman, Phys. Rev. B 64, 094507 (2001); L. Vidmar et al., Phys. Rev. Lett. 103, 186401 (2009).

[18] A. S. Alexandrov and A. F. Andreev, Europhys. Lett. 54, 373 (2001).

[19] A. S. Alexandrov, Physica C (Amsterdam) 305, 46 (1998).

[20] A. S. Alexandrov and K. Reynolds, Phys. Rev. B 76, 132506 (2007).

[21] I. Bozovic et al., Nature (London) 422, 873 (2003).

[22] H. Won and K. Maki, Phys. Rev. B 49, 1397 (1994).

[23] A. S. Alexandrov et al., Phys. Rev. Lett. 77, 4796 (1996).

[24] S. O. Katterwe et al., Phys. Rev. Lett. 101, 087003 (2008).

[25] S. Kaneko et al., Surf. Sci. 438, 353 (1999); A. Sugimoto et al., Physica C (Amsterdam) 412-414, 270 (2004); S. H. Pan et al., Phys. Rev. Lett. 85, 1536 (2000); K. McElroy et al., Phys. Rev. Lett. 94, 197005 (2005); Y. Kohsaka et al., Physica C (Amsterdam) 388-389, 283 (2003); T. Hanaguri et al., Nature (London) 430, 1001 (2004).

[26] Ruihua He and Z.X. Shen (personal communication). 